Culture et histoire dans l'espace roman

$6 \mid 2011$

Figures du pouvoir dans la littérature hispanoaméricaine

\title{
Le double sujet du pouvoir et la politique de la mémoire chez Ricardo Feierstein
}

"Olvidarse de quién era uno para poder sobrevivir »

\section{Martina Stemberger}

\section{OpenEdition}

Journals

Édition électronique

URL : https://journals.openedition.org/cher/9735

DOI : $10.4000 /$ cher.9735

ISSN : 2803-5992

\section{Éditeur}

Presses universitaires de Strasbourg

\section{Édition imprimée}

Date de publication : 30 juin 2011

ISBN : 978-2-35410-033-9

ISSN : 1968-035X

Référence électronique

Martina Stemberger, «Le double sujet du pouvoir et la politique de la mémoire chez Ricardo

Feierstein », reCHERches [En ligne], 6 | 2011, mis en ligne le 17 décembre 2021, consulté le 24 janvier

2022. URL : http://journals.openedition.org/cher/9735; DOI : https://doi.org/10.4000/cher.9735

\section{(c) (1) (2)(2)}

Ce(tte) œuvre est mise à disposition selon les termes de la Licence Creative Commons Attribution Pas d'Utilisation Commerciale - Partage dans les Mêmes Conditions 4.0 International. 


\section{Le double sujet du pouvoir et la politique de la mémoire chez Ricardo Feierstein "Olvidarse de quién era uno para poder sobrevivir»}

Martina Stemberger

Université de Vienne

Buenos Aires, au carrefour de l'Avenida General Mosconi et de la
rue Campana, l'après-midi du 2 février 1983: David Schnaiderman, âgé de quarante ans, père de famille au chômage, se rend à un entretien d'embauche; en traversant à la hâte la rue, il glisse sous la pluie; son dossier de présentation, contenant toute la documentation d'une biographie précarisée («Curriculum, ¡bah! Toda mi vida»1), s'envole de toutes parts. Un instant après, légèrement blessé, assis sur le trottoir, il assiste, seul témoin, à un meurtre. Mais, résultat du choc subi, il est frappé d'amnésie totale concernant non seulement la scène du crime mais aussi sa propre identité. Sur l'injonction de la police et à l'aide d'une talking cure qui constituera une bonne partie du roman Mestizo, paru en 1988, il essaiera de retrouver sa mémoire. Mais la question classique du genre policier - «Whodunit?» (Alewyn 1998: 57) - est vite reléguée au second plan: au désarroi du psychiatre chargé de ce cas difficile, la thérapie antiamnésique qu'entame le protagoniste sort d'emblée du cadre de l'enquête criminologique; Schnaiderman, à la recherche de ses «racines», se livre avec passion à une récupération nostalgique de son passé familial.

Dans ce roman policier postmoderne, "doble búsqueda» (M 275) aux traits métalittéraires prononcés (ce récit d'une quête identitaire, déguisé

1 Ricardo Feierstein, Mestizo [dorénavant désigné comme M],16. 
comme novela policial, s'inscrit, bien sûr, dans la tradition intertextuelle de ce "double" genre en Amérique latine), la tentative d'éclairer un crime à l'aide de ce témoin amnésique à plus d'un point de vue abouti à une vaste anamnèse personnelle, culturelle et politique, impliquant plusieurs générations d'un roman de famille judéo-argentin. Ce n'est pas par hasard que Feierstein déploie son panorama d'une Argentine en transition à travers un protagoniste marginal à plus d'un titre (intellectuel de gauche, sociologue au chômage, descendant d'une famille pauvre d'immigrés, Juif ayant vécu plusieurs années dans un kibboutz israélien) et qui, aux yeux des "autochtones" autoproclamés, doit d'abord prouver son droit à l'appartenance à une imaginaire communauté argentine. Schnaiderman se livrera d'ailleurs à une régulière déconstruction d'une argentinidad mythifiée, esquissant une contre-histoire du pouvoir et de la violence, mettant en question la légitimité d'un pouvoir "criminel", depuis la découverte jusqu'aux dictatures militaires du XXe siècle.

La redéfinition radicale de la relation entre majorité et minorité, «a "minorization" of the majority and a "majorization" of the minority" (Aizenberg 2002: 35sq.) - les minoritaires, pris dans leur ensemble, constituant bel et bien la majorité paradoxale de l'humanité -, est une idée centrale chez Feierstein dont la philosophie politique est fondée sur l'idée d'un «equilibrio dialéctico» entre "una mayoría "centrada" y rutinaria y una minoría "descentrada" y rebelde", cette dernière apportant à une société «el fermento necesario para producir el cambio» (M 302). Pour Feierstein (dont l'œuvre se situe dans le contexte de la déconstruction postmoderne des grands récits, dans le contexte des discours minoritaires et des «littératures mineures», voir Aizenberg 1993, et 2002: 40sq.), il s'agit d'interroger les mythes fondateurs de la "nation" argentine (crisol de razas, "civilización vs barbarie», tierra de promisión des immigrés etc.) à partir de positions stratégiquement minoritaires, de jeter un regard délibérément «excentrique» sur cet «Extraño país, mi Argentina» (M 292).

En un mot, Mestizo est un texte éminemment politique; une réflexion complexe sur les ambivalences d'un pouvoir générateur et annihilateur d'identités, instance d'assujettissement au double sens du terme entre soumission et subjectivation (Butler 2001: 87) -, sur les rapports difficiles entre "pouvoir», "violence», «autorité», «force». Feierstein décrit minutieusement le potentiel pathogène du pouvoir dictatorial, les déformations du sujet dans « $[\mathrm{u}] \mathrm{n}$ clima de terror y espanto, todo el día, 
todos los días $»^{2}$, dans une société où presque tout le monde est suspect, où la mort court les rues (M 296). Avec une précision quasi clinique, il analyse les ravages de l'angoisse généralisée, les effets dévastateurs d'un régime imposant la paranoïa comme condition de survie (286).

Mestizo évoque toute une panoplie de réactions possibles face au régime de la peur, à la terreur de la disparition et de l'oubli; de la résistance violente en passant par le refoulement jusqu'à la psychose manifeste: il y a Teresa qui, après avoir été obligée d'identifier son frère assassiné, transformé en "un montón de carne sangrante», refuse de se souvenir, de parler politique(221); la fille de la victime du meurtre, "Leila alias Sabina alias la Turca» (271), personnage aux masques multiples, ancien membre d'un groupe de guérilla, maintenant professeur de danse; son frère Ahmed, membre d'un "grupo de choque" pro-palestinien aux ambitions terroristes, adepte d'un culte cynique du pouvoir et de la force, déniant toute possibilité de solidarité - ne fût-ce que stratégique - entre minoritaires: «Unos dominan a otros. [...] Se lucha por el poder, para dominar a otros, ser superior. Siempre ha sido así, en todo el mundo. La fuerza es lo que finalmente triunfa: todo el resto son construcciones intelectuales [...]» (318sq.). Mais il y a aussi León Piatigorsky, ami de jeunesse du protagoniste, ayant dûment mérité son sobriquet "pruebalotodo», qui se lance dans des aventures politiques, économiques, érotiques de plus en plus absurdes, en proie à une mégalomanie compensatrice, camouflant la peur et le désarroi; et surtout Ignacio, victime "idéale" depuis son enfance, dont la personnalité cède très vite aux fantômes de "resentimiento, autodestrucción, locura" (340). Ignacio - n'ayant vécu que par procuration, lié par un rapport ambigu à León, dont il est le double négatif et misérable, le «delegado personal» (167) poussant son "rôle" jusqu'au meurtre - illustre la fragmentation pathologique d'un sujet qui, terrorisé, procède à son auto-annihilation.

Obsédé par la peur, Ignacio, «Un muerto en vida» (323), décide de brûler sa bibliothèque, de la faire disparaitre dans un autodafé très symbolique: «La biblioteca es el esqueleto de la memoria. Hubo que hacerla desaparecer, así como la dictadura secuestraba y desaparecía a las personas. Olvidarse de quién era uno para poder sobrevivir» (260). David Schnaiderman, lui, a refusé de brûler ses livres; mais il s'est vu obligé de les disperser, de les cacher, tout comme son moi fragmenté, dans les endroits les plus divers. La bibliothèque comme métaphore (très borgésienne) de la mémoire, de

2 Ricardo Feierstein, La logia del umbral [dorénavant désigné comme L], 286. 
l'identité peut être considérée comme un leitmotiv de l'œuvre feiersteinienne. Dans La logia del umbral, c'est Marcelo Schvel qui, pendant les années de la dictature, a enfermé «todos los libros sospechosos» ( $L$ 286) dans le cabinet psychanalytique de sa sœur. Pour lui aussi, la bibliothèque cachée équivaut à une "amputation» temporaire d'une partie de son propre moi ("Quise sobrevivir. Esconder los libros y a mí mismo con ellos», 289) qu’il essaiera de réincorporer, une fois le danger passé.

Au moment de sa cure narrative, Schnaiderman (fidèle à son nom, expert en matière de "hyphologie » identitaire, "cut-and-paste man », Stavans 2000), hanté par l'obsession de sauver sa «bibliothèque» au sens concret et métaphorique du terme, voue un véritable culte à la chose écrite. Ce n'est pas par hasard que le personnage-clé dans son entreprise de "recollection", le grand-père mythique Moishe Búrej, tailleur-philosophe, «un adelantado de los psicoanalistas lacanianos» ( $M$ 227), «autoridad moral» reconnue même par la population non-juive de son village (47) - bref: sujet «résistant» -, est à son tour comparé à "un archivo que caminaba" (48), dont l'accès était défendu au protagoniste même du vivant du vieillard, faute d'une langue commune. Schnaiderman en vient à regretter amèrement d'avoir perdu la langue de ses ancêtres et tout l'héritage culturel qu'elle véhiculait: «Estoy seguro que, de haber comprendido entonces, todo me sería mucho más sencillo ahora» ( $M$ 36). Si la bibliothèque - même dispersée - apparaît comme foyer secret de la résistance du sujet, ce dernier se conçoit en termes de palimpseste, espèce de Wunderblock freudien; pour Schnaiderman, il s'agit de rendre visibles et lisibles les traces mémorielles de la scène du crime comme de son passé perdu (311).

Le choc amnésique et le travail an-amnésique du protagoniste se situent dans le cadre d'une société émergeant de la terreur, traumatisée par "[a]ños de muertes y torturas y desapariciones" (275). Schnaiderman, "symptôme" surdéterminé, apparaît comme victime tardive d'une "amnésie" généralisée; la scène du meurtre ne fait que donner le coup final à une mémoire déjà minée par l'oubli: «Ese debió ser el comienzo de mi amnesia. [...] Una vez me detuvieron [...] Quise olvidarlo, lo negué. Allí comencé a perder la memoria. [...] Pero sobreviví...» (261). L’oubli, dans ce contexte, apparaît comme un réflexe de défense élémentaire («El cerebro dice "basta", se preserva», 269), comme stratégie d'auto-exculpation («Por eso resulta difícil haber sobrevivido. No queremos recordar para no sentirnos culpables», 262). Face à la terreur de l'oubli et de la disparition, la «mémoire active» apparaît comme acte de résistance; comme devoir aussi de témoigner au nom des disparus (290). 
Mais les relations entre le pouvoir - qui n'est pas «une institution» ni "une structure» ni "une certaine puissance dont certains seraient dotés», mais «le nom qu'on prête à une situation stratégique complexe dans une société donnée», selon la célèbre définition «nominaliste» de Foucault (1976: 123) - et «ses» sujets ne sont jamais si simples que cela. Mestizo constitue une réflexion sur les mécanismes du double "assujettissement" bien au-delà des contextes politiques. Le roman contient toute une série de mini-scènes, illustrant la dynamique complexe du pouvoir dans divers microcosmes sociaux. Est exemplaire, à cet égard, la description d'un cours de ballet que fréquente la fille de Schnaiderman; le narrateur observe la secrète chorégraphie de l'inclusion et de l'exclusion, la répression impitoyable exercée par des petites filles qui ne pardonnent rien ( $M$ 258). Toute cette scène est encadrée par une discussion politique entre les spectateurs adultes; danse des enfants et discussion des parents, orchestrées en contrepoint, se reflétant mutuellement.

Le roman propose une vue nuancée sur un pouvoir politique s'autodiscréditant par la violence; mais d'un autre côté, sur fond des biographies de ces éternels minoritaires, "survivants" malgré tout et malgré tous, la violence défensive est représentée comme légitime, voire nécessaire: c'est aussi une leçon que le protagoniste tirera de son passé familial ( $M$ 46). Ce qui était vrai pour Moishe Búrej dans son shtetl de la zone frontalière russo-polonaise («Éramos una tierra de nadie, de frontera», 59), face aux persécutions antisémites officielles et officieuses, face à la terreur du quotidien, l'est aussi pour David et León, agressés comme "rusitos de mierda» dans une rue de Buenos Aires (214). À partir de scènes pareilles, Mestizo analyse les implications de l'«interpellation» (Butler 2001: 91-92, 101-123, ou 2006: 44sq.), les ambivalences de l'expérience « to be called a name» (Butler 2006: 9sq.). Dans plusieurs de ses œuvres, Feierstein réfléchit sur la problématique du nom "étranger" stigmatisant (motif important dans la littérature judéolatino-américaine en général); dans Mestizo, cette réflexion culmine dans un chapitre au titre significatif Aventuras de un apellido (137sq.).

Que devient la scène classique de l'interpellation, selon Althusser, dans l'Argentine des années du Proceso? Mestizo met en lumière les engrenages du pouvoir, les mécanismes de domination dans une société qui produit des sujets normés, conformistes, dénués de passé, de mémoire, des sujets disciplinés, malléables et rigides en même temps, désindividualisés, incorporant «una nueva imagen de hombres neutros, elusivos, algo estúpidos. Tal como quería el régimen» (296). Des sujets qui apprennent 
à s'auto-coloniser (Butler 2001: 99), à s'auto-discipliner, à "se disparaittre" avant même d'être touchés par la terreur.

Mestizo montre aussi comment ce "dressage" (se) passe par le corps, comment une société crée des corps dociles. C'est encore à travers l'imagerie du corps - surtout de la danse, entre discipline imposée et extase du mouvement libre - que le roman illustre l'ambivalence d'un dressage qui peut aussi être "retourné", dans le sens de l'«enabling violation» (Gayatri C. Spivak, voir Landry/ MacLean 1996: 19). La transition vers une nouvelle société démocratique est métaphorisée comme un événement corporel, comme une libération physique: "La esperanza es una danza» ( $M$ 343). Le corps, objet de pratiques disciplinaires, mais aussi source d'autonomie et de bonheur, la mémoire corporelle jouent un rôle-clé dans l'entreprise de ré-identification du protagoniste, redécouvrant, par exemple, ses idiosyncrasies culinaires, obscur héritage, elles aussi, d'un passé familial reconquis coup sur coup.

Mestizo, réflexion sur la continuelle et mutuelle formation du pouvoir et de ses sujets, soulève la question de savoir quelle nouvelle subjectivité serait donc susceptible de fonder - et d'être fondée par - une nouvelle société démocratique. Feierstein, dans sa fiction littéraire comme dans son œuvre essayistique, problématise l'aspect "idéologique», voire "criminel» de toute identification dont parle Vilém Flusser (2007: 120 et 2000: 101); il développe sa propre version du concept controversé du mestizaje, conçu comme modèle d'une identité juive et latino-américaine, identité performative, polyphone, fondée sur les «twin axes» (Aizenberg 2002: 35) du pluralisme et de la "supervivencia», l'art de "survivre» dans des conditions historiques des plus difficiles, unissant Juifs et Latino-Américains non-juifs, «mestizos y sobrevivientes» (M 360). Il ne s'agit plus de fondre une minorité persécutée - la minorité par excellence - dans le creuset de la majorité, mais de revaloriser l'expérience juive comme modèle d'une identité latino-américaine, positivement marginale (idée qui se trouve déjà chez Borges dans El escritor argentino y la tradición, voir Borges 2007: 200sq.).

Sur fond de cette re-conceptualisation du mestizaje, Feierstein procède à une «déracialisation» systématique du judaïsme. Au cours de ses recherches identitaires, Schnaiderman se voit confronté à des détails surprenants sur ses origines; ainsi, il apprend qu'il est, selon toute probabilité, un descendant des Khazars mythiques ( $M$ 225sq.), donc non pas «juif» sémite selon des catégories strictement raciales. Cette découverte inattendue donne lieu à une 
méditation sur la définition difficile d'une identité "juive"; le protagoniste en arrive à la conclusion que «el judaísmo es un hecho cultural y antropológico, antes que étnico o religioso » (226). Bref: «on ne naît pas Juif, on le devient» - et pas exclusivement par le regard et le discours de l'antisémite, dans le sens de Sartre; on «se fait» plutôt Juif (226) par toute une série d'actes minuscules, quotidiennement répétés (à propos du judaïsme comme mode de vie «rituel», voir Flusser 2000 : 94).

Ette souligne, à juste titre, que le concept du mestizaje, au-delà de son histoire politiquement plus qu'incorrecte, est pourvu d'un certain potentiel «totalitaire», d'une tendance "centralisatrice» qui trouve toujours quelque chose à «dédifférencier», à "fusionner» (Ette 2001: 519). Toumson, dans sa Mythologie du métissage, insiste de même sur la problématique d'une "psychologie fusionnelle», impliquant une "négation de l'altérité» (Toumson 1998: 260, cit. Ette 2001: 500). Qu'en est-il chez Feierstein? À la fin de Mestizo, une certaine extase de la «fusion», d'un «moi» enfin intégré à un collectif triomphal semble indéniable. Mais Feierstein n'est pas aveugle aux dangers du mestizaje et des tentations totalitaires de la première personne du pluriel: la réflexion critique dans Mestizo porte aussi sur les ambivalences du collectif, même démocratique et révolutionnaire.

Après un match de football dont leur club est sorti gagnant, Schnaiderman et son fils, minoritaires "héréditaires", s'avouent déconcertés par la sensation inouie de faire partie d'une majorité; la question du collectif revient dans leurs discussions lorsqu'il s'agit de participer aux manifestations pro-démocratiques du printemps 1983; le fils explique au père qu'il est impensable de s'y rendre, comme ce dernier, pas encore libéré de ses réflexes d'individualiste, avait l'intention de le faire, tout seul ou en couple; qu'il faut, ne fût-ce que pour des raisons de sécurité, faire partie d'un groupe, il n'a que l'embarras du choix entre «las Madres de Plaza de Mayo, el Movimiento Judío por los Derechos Humanos, algún partido político» (M 330). En un mot, narrateur(s) et personnages sont très conscients des ambivalences $\mathrm{du}$ collectif, d'une nouvelle démocratie aux traits quelquefois un peu grégaires. Ce qui sauve le mestizaje feiersteinien du danger totalitaire, c'est la polyphonie conservée au sein de l'euphorie fusionnelle même; la conception de l'identité mestizo que préconise l'œuvre de Feierstein se veut multiple, kaléidoscopique, plurielle.

L'intérêt particulier de Mestizo est dû au fait que la quête identitaire, l'exploration d'une subjectivité polyphone ne se limite pas au plan du contenu; elle se reflète dans la texture d'un roman où la langue elle-même 
devient «a zone of hybridity» (Aizenberg 2002: 37), où le mestizaje est aussi un fait linguistique (à propos de certains passages, Aizenberg parle de «Spanyish», 2002: 38), où le yiddish, langue minoritaire paradigmatique, vient subvertir et enrichir l'espagnol en tant que langue majoritaire (40sq.).

Au-delà de la réflexion sur langues majoritaires et minoritaires, l'œuvre de Feierstein esquisse une véritable «politique de la narration». Mestizo est aussi un roman sur la philosophie de la voix et du mode narratifs, du pronom personnel, d'une subjectivité renégociée entre les conventions de la personne grammaticale, entre Raíces en YO, Tronco y TU, Extremidades con EL et, enfin, Árbol, NOSOTROS - avec, en épigraphe, une phrase du psycholinguiste A.A. Leontiev, résumant la "philosophie pronominale» du texte: «Los pronombres son un espejo en el que se refleja el sistema de relaciones sociales» (M 345).

Mestizo - œuvre hybride, "montage" d'extraits de journaux, de bandes dessinées et surtout de photographies qui, douées de leur propre "narrativité visuelle» (Bal 2006: 322), sont beaucoup plus que de simples «illustrations» - pousse la polyphonie à l'extrême: dès les premières séances de la cure narrative du protagoniste, transformé tout entier en corps de résonance, les lecteurs ont affaire à une explosion de voix fantomales, surgissant du passé familial, à un chaos de narrateurs à la première personne qui ne cessent de se disputer l'autorité narrative, le contrôle sur l'histoire à reconstruire. Schnaiderman libéré par le choc polyphonique de la première partie, de la "prison" idéologique d'un je problématique, continue sa recherche identitaire à la deuxième personne (ce déplacement pronominal réfléchissant aussi un développement personnel), adoptant ensuite le point de vue de la troisième personne pour se joindre enfin à un "nous" kaléidoscopique (Aizenberg 2002: 37, 41). Bref: tout ce roman s'applique à déconstruire la personne comme entité stable et univoque. Le sujet narratif apparaît comme polyphonie joyeuse, chaotique, plus ou moins "domestiquée" par les conventions de l'identité et de l'usage du pronom à la première personne, générateur d'une cohérence précaire, mais aussi « un opérateur de métalepse » (Genette 2004: 110).

Mestizo finit sur une tonalité optimiste, avec une danse sur la mélodie de l'espoir. Qu'en est-il une quinzaine d'années après, au début du XXIe siècle? Pour voir l'évolution ultérieure de la vision feiersteinienne d'une "Argentina 1983» (M 343), je voudrais analyser brièvement un autre roman du même auteur, publié en 2001. Tout comme Mestizo, La logia del Umbral, texte orchestré avec virtuosité, "hybridisé" aussi sur le plan linguistique, mise 
sur des éléments du genre policier, les intégrant à la structure ludique d'un roman conçu comme «jeu» identitaire.

Nous retrouvons une famille judéo-argentine comme protagoniste collectif, organisme polyphone, même si, à la différence de Mestizo, toutes les voix narratives sont proprement "encadrées". Les personnages principaux sont encore des marginaux paradigmatiques, ornés du nom de famille très symbolique de «Schvel», le "seuil» en yiddish, d'où le titre du roman. La logia del umbral éponyme n'est rien moins que le concept utopique d'une communauté des hommes et des femmes du seuil, de l'entre-deux; d'une union de tous les minoritaires qui, enfin réunis, seraient capables de réclamer leurs droits de majorité paradoxale. Mariano Moisés (qui insiste sur son droit à l'hybridité, sur son droit de ne pas choisir: "Mi plan es integral, mestizo, creativo. No renuncio a nada [...] Quiero todo, lo argentino y lo judío", $L$ 245) conçoit son nom de famille comme une véritable «mission", non sans une certaine dose d'ironie, la mission paradoxale de démonter toutes les missions, de déconstruire toutes les certitudes, de remplacer les grands récits par des narrations consciemment minoritaires: « esta "logia del umbral" hace honor a su denominación: siempre nuestro relato es parcial, limitado, sin generalizaciones indebidas» (290).

À travers cette famille "liminale», chargée de la mission ludique de «liminaliser» le monde, est renégociée la perspective d'une nouvelle identité argentine, juive, latino-américaine. L'histoire argentine est systématiquement mise en relation avec l'histoire juive, Feierstein soulignant encore, sans relativiser toutefois l'expérience de la Shoah, les affinités entre Juifs et Argentins, les deux "survivants" paradigmatiques (topos désormais établi dans le discours politico-médiatique). En même temps, l'image d'une Argentine démocratique, pluraliste, esquissée à la fin de Mestizo (qui coïncide avec la fin de la dictature militaire) est fondamentalement remise en question. Il est significatif que Feierstein, dans son évocation des événements traumatiques des années 90 , ait recours au mythe du gaucho judío, procédant à une "révision" du texte canonique de Gerchunoff. Ainsi, la scène classique de l'arrivée des nouveaux immigrés dans leur Terre promise («Llegada de inmigrantes», Gerchunoff 2007: 57sq.) subit une modification considérable.

Presque un siècle plus tard, Mariano Schvel, ayant repris le prénom de «Moisés» (en hommage à son ancêtre Moishe, père fondateur des Schvel argentins, assassiné par un gaucho), s'apprête à accomplir, "como auténtico descendiente de esos gauchos judíos que soñaron con la tierra libre de Sión 
en estas latitudes» ( $L$ 245), un rituel d'intégration symbolique. À partir de Moisesville, il veut refaire le parcours du judaïsme argentin, pour arriver enfin, des marges du pays et de la société, à son cœur métaphorique même, «la Plaza de Mayo [...] ese lugar de partida y llegada de la nacionalidad». Dans la scène d'ouverture du roman, nous voyons donc ce personnage, déguisé en gaucho judío contemporain, traverser à cheval le centre de Buenos Aires, muni d'une caisse remplie de terre ( «la tierra de inmigrantes [...] fecundada con sangre y esfuerzo, rezos y trabajo duro, nacimientos y muertes», 245) qu'il veut déposer à côté de l'héritage secret de Perón. Le jeune cavalier est attendu par toute la "logia del umbral», pour célébrer le grand événement: «El fin del juego de la integración judía en la Argentina» (290), la preuve que l'idéal de «la fraternidad argentina, mestiza e integradora» s'est, malgré tout, avéré possible (291).

Le long et difficile parcours de l'intégration juive en Argentine semble enfin réussi, cette matinée du 18 juillet 1994, date que nous, lecteurs, savons d'avance funeste. Le gaucho judío postmoderne, conscient à la fois de l'importance et de l'ironie de son rôle, n'atteindra jamais son but; il tombe tout droit dans l'attentat de l'AMIA. Tout comme David Schnaiderman, Mariano Schvel se transforme en témoin traumatisé au coin d'une rue de Buenos Aires; mais témoin d'un crime d'une tout autre envergure, s'imposant le devoir de fixer dans sa mémoire l'inconcevable ( Testigo judío en una calle argentina. [...] me he impuesto no desviar la mirada», 296), tandis que, devant ses yeux, la danse joyeuse de l'espérance, évoquée dans Mestizo, dégénère en «danse» perverse d'une «ráfaga de metralleta» (296).

Nous sommes loin de l'optimisme qui avait marqué la vision d'une Argentine nouvelle à la fin de Mestizo. Toutefois, la «logia del umbral» ne renonce pas définitivementà son rêve d'une société ouverte et plurielle; le roman, fidèle à sa structure ludique, opposé au sérieux meurtrier des fanatismes idéologiques, finit sur un épilogue, résumant «El sueño compartido de la Logia del Umbral (interpretado por Myriam)», la psychanalyste de la famille (299sq.). Cette interprétation de rêves, d'inspiration freudienne et deleuzienne, aboutit à une question toujours sans réponse: “¿Cómo terminará un "Juego de la Integración" donde no sólo participamos nosotros, sino también los otros?» (304). 


\section{Bibliographie}

Aizenberg, Edna, 1993, «Jewish Identity, Pluralism, and Survival: Feierstein's Mestizo as Minority Discourse », in Robert DiAntonio et Nora Glickman (ss dir.), Tradition and Innovation. Reflections on Latin American Jewish Writing, Albany (NY), SUNY, p. 107-114.

Aizenberg, Edna, 2002, «Ricardo Feierstein's Mestizo: Kaleidoscopic Identities and Human Rights", in Aizenberg, Books and Bombs in Buenos Aires: Borges, Gerchunoff, and Argentine-Jewish Writing, Hanover (NH), UP of New England, p.36-42.

Alewyn, Richard, 1998, "Anatomie des Detektivromans», in Jochen Vogt (ss dir.),

Der Kriminalroman: Poetik. Theorie. Geschichte, München, Fink, p. 52-72.

Arendt, Hannah, 2008, Macht und Gewalt, München, Piper.

Bal, Mieke, 2006, Kulturanalyse, Frankfurt a. M., Suhrkamp.

Borges, Jorge Luis, 2007, Discusión, Madrid, Alianza, coll. «Biblioteca Borges».

Butler, Judith, 2001, Psyche der Macht. Das Subjekt der Unterwerfung, Frankfurt a. M., Suhrkamp.

Butler, Judith, 2006, Haß spricht. Zur Politik des Performativen, Frankfurt a. M., Suhrkamp.

Eglau, Victoria et Peşman, Gözde, 2010, «Acercamiento literario a la dictadura militar en Argentina», 25.09.2010 (disponible sur le site http://www.dw-world. de/dw/article/0,,6033177,00.html).

Ette, Ottmar, 2001, Literatur in Bewegung. Raum und Dynamik grenzüberschreitenden Schreibens in Europa und Amerika, Weilerswist, Velbrück.

Feierstein, Ricardo, 1984, Sinfonía inocente, Buenos Aires, Pardés.

Feierstein, Ricardo, 1994, Mestizo, Buenos Aires, Planeta. [= M]

Feierstein, Ricardo (ss dir.), 1998, Los mejores relatos con gauchos judíos. De Alberto Gerchunoff a Marcos Aguinis, Buenos Aires, Ameghino.

Feierstein, Ricardo (ss dir.), 2000, Alberto Gerchunoff, judío y argentino: viaje temático desde "Los gauchos judíos» (1910) hasta sus últimos textos (1950) y visión crítica, Buenos Aires, Milá.

Feierstein, Ricardo, 2001, La logia del umbral, Buenos Aires, Galerna. [= L]

Flusser, Vilém, 2000, Jude sein. Essays, Briefe, Fiktionen, Berlin/Wien, Philo.

Flusser, Vilém, 2007, Von der Freiheit des Migranten. Einsprüche gegen den Nationalismus, Berlin/Wien, EVA.

Foster, David William, 2000, «Ricardo Feierstein. Mestizo [Review]», World Literature Today, vol. 74, n 4 , p. 899.

Foucault, Michel, 1976, La volonté de savoir (Histoire de la sexualité I), Paris, Gallimard.

Genette, Gérard, 2004, Métalepse, Paris, Seuil.

Gerchunoff, Alberto, 2007, Los gauchos judíos. El hombre que habló en la Sorbona [1926], Buenos Aires, Colihue / Biblioteca Nacional, coll. «Los Raros». 
Landry, Donna et MacLean, Gerald (ss dir.), 1996, The Spivak Reader. Selected Works of Gayatri Chakravorty Spivak, New York/London, Routledge.

Ran, Amalia, 2009, "Nuestra Shoá: Dictaduras, Holocausto y represión en tres novelas judeorioplatenses », Letras hispanas, $n^{\circ} 6 / 1,2009$, p. 17-28.

Stavans, Ilan, 2000, "Introduction», in Ricardo Feierstein, Mestizo. A novel, Albuquerque, U of New Mexico P, p. IX-XV.

Toumson, Roger, 1998, Mythologie du métissage, Paris, PUF.

Verdevoye, Paul, 1991, "Le métissage judéo-argentin dans la trilogie Sinfonía inocente, de Ricardo Feierstein", in Claude Cymerman (ss dir.), Le roman hispano-américain des années 80. Actes du colloque de Rouen 26-28 avril 1990, Les Cahiers du CRIAR n¹1, Publications de l'Université de Rouen $\mathrm{n}^{\circ} 166$, p. 63-70. 